\title{
A Rectenna as Energy Source for Wireless Sensor Nodes
}

\author{
Andry Contreras*, Leonardo Steinfeld ${ }^{\dagger}$, Mariana Siniscalchi ${ }^{\dagger}$, Javier Schandy ${ }^{\dagger}$ and Benigno Rodríguez ${ }^{\ddagger}$ \\ * Dept. of Circuits and Communications, Universidad del Zulia, Maracaibo, Venezuela \\ Email: acontreras@fing.luz.edu.ve \\ $\dagger$ Dept. of Electronics, Universidad de la República, Montevideo, Uruguay \\ Email: leo@fing.edu.uy, msiniscalchi@fing.edu.uy, jschandy@fing.edu.uy \\ $\ddagger$ Dept. of Telecommunications, Universidad de la República, Montevideo, Uruguay \\ Email: benigno@fing.edu.uy
}

\begin{abstract}
This paper presents the feasibility assessment for the adoption of a rectenna with basic structure elements as energy source in wireless sensor networks (WSN) nodes. For that matter, a rectenna that harvests energy from $2.45 \mathrm{GHz}$ was designed by the application of a tuning technique on the dimensions of its elements. In the design, the CST Microwave Studio and a SPICE based software were used. The DC-DC boost converter was selected among several commercial options. The limitations of the rectenna were evaluated using the power consumption of different nodes. Results show that the total maximum conversion efficiency for the rectenna was $34.08 \%$ at $20 \mathrm{dBm}$. The distance ranges between the designed rectenna and the wireless energy source were from $0.03 \mathrm{~m}$ to $0.20 \mathrm{~m}$ in the indoor scenario and from $0.26 \mathrm{~m}$ to $2.03 \mathrm{~m}$ in the outdoor scenario. The rectenna can supply the required power for the nodes CC2538 and CC1350 in the full range of distances, but for the nodes $\mathrm{Z1}$ and Sky the range of distances is reduced.
\end{abstract}

Index Terms-Rectennas, Energy Harvesting, DC-DC Boost Converter, Wireless Sensor Networks, Wi-Fi.

\section{INTRODUCTION}

Wireless sensor networks (WSN) consist of the interconnection of nodes, containing sensors and actuators, through wireless communication for various applications. These networks are self configurable, low-cost and low-energy consuming. Their purpose is to monitor physical and environmental conditions while sharing data cooperatively towards a main node in, for example, convergecast applications. The main node processes the information and executes a specific series of actions. Each node is constituted by an energy source, a microcontroller, a radio and input/output ports for sensors and actuators [1].

The implementation of WSN has challenges, such as their damage tolerance in dangerous environments, the restriction in the number of sensors and actuators which increments the energy consumption, the interference in communications between nodes due to the operating frequency, and the energy consumption of the radio. Most of these challenges are related to limitations in energy resources [2].

The lifetime of the nodes is incremented using sources such as solar, wind, thermal, vibrations and electromagnetic energy. Photovoltaic systems are the most used renewable energy sources for WSN. Nevertheless, their efficiency depends on the solar irradiance at the geographic location where they are installed and their implementation can increase the costs [3].

An alternative low-cost renewable energy source are the rectennas, which are devices that comprise an antenna and a rectifier to capture and harvest electromagnetic waves from microwave band and to transform them into electrical energy for its utilization [4], [5], i.e. they are a wireless energy source. Therefore, the energy is harvested from the propagating waves present in the environment, such as urban or offices.

In this research, the feasibility for the adoption of a rectenna as energy source in WSN nodes to increase their lifetime was assessed. Although the design of rectennas has advanced progressively in recent years, in this paper a low-cost rectenna with basic structure elements was evaluated. This is considered as an starting point for future research that will incorporate state-of-the-art modifications to the basic structure in order to optimize the performance of the rectenna.

The elements of the rectenna were designed through a tuning technique and the power that can be harvested from the $2.45 \mathrm{GHz}$ band was determined. Furthermore, the power consumption of several nodes sending messages was evaluated, to analyze the performance of the rectenna and its limitations in WSN applications.

\section{Design OF THE RECTENNA}

In this work, the energy source is a rectenna that enables the harvesting and utilization of electromagnetic waves, designed, simulated and tuned by the authors, whose elements are shown in Fig. 1. The antenna receives the electromagnetic waves and transform them into AC signals. This is connected to the LPF, that improves the impedance coupling and eliminates the harmonics generated by the rectifier [6]. The rectifier transforms the AC voltage signal into DC and conducts it to the DC-DC boost converter that keeps the voltage at a functional level for the node. This converter can regulate the voltage from an input within a range that depends on the model and the integrated circuit configuration [7].

This energy source harvests electromagnetic energy at the design frequency of $2.45 \mathrm{GHz}$ because of its massive use, with low-cost and simple fabrication elements. For that, the 


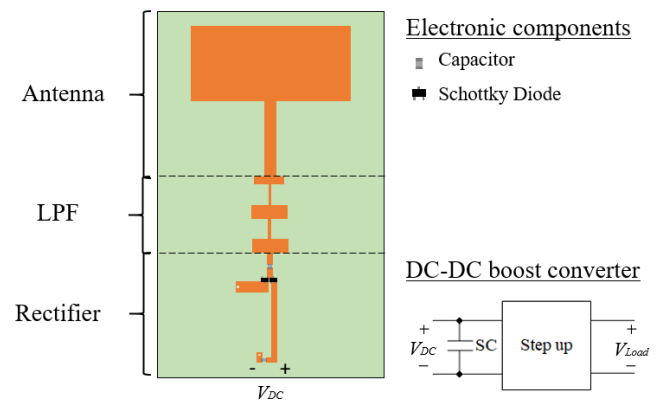

Fig. 1. Layout of the rectenna that comprises antenna, LPF and rectifier with the circuit diagram of the DC-DC boost converter.

rectenna was designed on FR-4 substrate, which has a dielectric constant $\epsilon_{r}=4.4$, a dielectric loss tangent $\tan \delta=0.02$, and a thickness equal to $1.6 \mathrm{~mm}$.

A tuning technique was employed by means of adjusting the dimensions of each one of the elements, with a resolution of $1 \mathrm{~mm}$. For that process, the antenna and the filter behavior were simulated using the CST Microwave Studio software [8], while for the rectifier circuit a high frequency circuit simulator based on SPICE was employed.

Antenna: A rectangular printed microstrip type with feeding microstrip was designed. The simulation results at $2.45 \mathrm{GHz}$ for the tuned antenna were an $S_{11}$ magnitude of $-24.12 \mathrm{~dB}$ (Fig. 2), a standing wave ratio (SWR) of 1.133 , an impedance of 50.853-j6.072 $\Omega$ and a gain of $3.38 \mathrm{dBi}$ on $\mathrm{H}$ plane with $\phi=0^{\circ}$, obtaining the directional radiation pattern presented in Fig. 3.

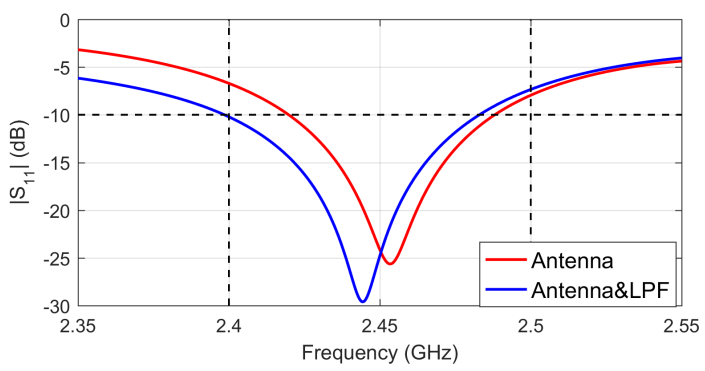

Fig. 2. Simulated $S_{11}$ magnitude for the antenna and for the set of antenna \& LPF.
LPF: A LPF of stepped impedance type was designed. The tuned set of antenna and LPF was simulated obtaining an $S_{11}$ magnitude equal to $-24.79 \mathrm{~dB}$ (Fig. 2), which represents an improvement for the design frequency. Also, this process enhanced the matching impedance between the antenna and the filter, as shown in Fig. 2.

Rectifier: The design of this element was a voltage doubler with a single stage configuration, with a Schottky diode HSMS-2820. The RF to DC conversion efficiency and the DC voltage of the energy source simulated as functions of the input power as shown Fig. 4. The maximum value of RF to DC conversion efficiency was $40.09 \%$ and the maximum rectification voltage was $6.1 \mathrm{~V}$, both for a power of $20 \mathrm{dBm}$ at the input of the LPF.

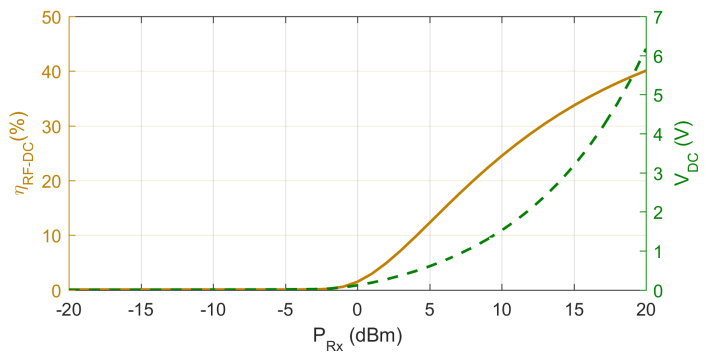

Fig. 4. Simulated conversion efficiency and voltage at the output of the combination of antenna, LPF and rectifier as functions of $P_{R x}$.

The RF to DC conversion efficiency is determined with (1) and it takes into consideration the LPF and the rectifier efficiencies, where $P_{D C}$ is the rectified electric power and $P_{R x}$ is the power received taking into account the gain of the rectenna.

$$
\eta_{R F-D C}=\frac{P_{D C}}{P_{R x}} 100 \%
$$

DC-DC boost converter: Regarding the design of this element, a supercapacitor (SC) was employed at the input in order to store the harvested energy, and a step-up converter was connected to hold the voltage in the output during the operation of the nodes. Different step-up converters are evaluated in Section III for their application in WSN.

\section{CONDITIONS FOR THE APPLICATION OF THE DESIGNED RECTENNA FOR WSN}

In order to supply the harvested energy to the nodes of the WSN there are a series of conditions that must be fulfilled. The first condition is expressed in (2), where the left-hand side of the equation is the power harvested by the rectenna, $\eta_{\text {total }}$ is the rectenna total conversion efficiency (it depends on the RF to DC conversion and the selected DC-DC boost converter efficiency values) and $P_{\text {node }}^{\min }$ is the power consumed by the communication and processing unit. If this condition is accomplished, the node will be permanently operative because the source supplies $100 \%$ of the energy for its utilization.

$$
\eta_{\text {total }} \cdot P_{R x} \geq P_{\text {node }}^{\text {min }}
$$


The second condition is that the operating voltage of the communication and processing unit must have fixed values. Therefore, a DC-DC boost converter is included in the design of the energy source, even when the input voltage varies, as long as it is equal to or higher than the start-up voltage, that is (3) where $V_{D C}$ is the rectified voltage and $V_{D C}^{\text {min }}$ is the startup voltage. The selected operating voltage was $3.3 \mathrm{~V}$ because it is a commonly employed operating voltage for nodes.

$$
V_{D C} \geq V_{D C}^{\min }
$$

In this work, the implementation of several types of DCDC boost converters is evaluated, and their characteristics and application results are shown in Table $\mathrm{I}$, where $P_{R x}^{\min }$ is the minimum input power required by the rectenna to supply $V_{D C}^{\min }$, taking into account the gain of the tuned antenna. It is noted that the total conversion efficiency and the power harvested with the DC-DC boost converters are lower than the ones obtained by the rectenna without them. This is the cost of maintaining the voltage level at a fixed value.

Considering the characteristics of the selected converters, the maximum power harvested by the rectenna with a $20 \mathrm{dBm}$ input power is between $32 \mathrm{~mW}$ and $36 \mathrm{~mW}$, as shown in Fig. 5. The values of the power harvested for the evaluated converters are close to one another, therefore this characteristic is not determinant for the selection of a particular step-up converter model.

TABLE I

CHARACTERISTICS AND APPLICATION RESULTS OF THE SELECTED DC-DC BOOST CONVERTERS WITH AN OUTPUT VOLTAGE OF $3.3 \mathrm{~V}$.

\begin{tabular}{|c|c|c|c|c|}
\hline \multicolumn{2}{|c|}{ DC-DC boost converter } & \multicolumn{4}{|c|}{ Rectenna } \\
\hline $\begin{array}{c}\text { Part } \\
\text { name }\end{array}$ & $\begin{array}{c}V_{D C}^{\min } \\
(\boldsymbol{m} \boldsymbol{V})\end{array}$ & $\begin{array}{c}P_{R x}^{\min } \\
(\mathbf{d B m})\end{array}$ & $\begin{array}{c}\eta_{\text {total }}(\boldsymbol{\%}) \\
@ P_{R x}^{\min }\end{array}$ & $\begin{array}{c}\eta_{\text {total }} \mathbf{( \% )} \\
@ \mathbf{2 0} \mathbf{~} \mathbf{B m}\end{array}$ \\
\hline ADP5090 [9] & 380 & 3 & 5.77 & 32.07 \\
\hline TPS61202 [10] & 500 & 5 & 10.44 & 32.87 \\
\hline LTC3105 [11] & 250 & 2 & 4.29 & 34.08 \\
\hline MAX1675 [12] & 900 & 7 & 15.70 & 36.04 \\
\hline
\end{tabular}

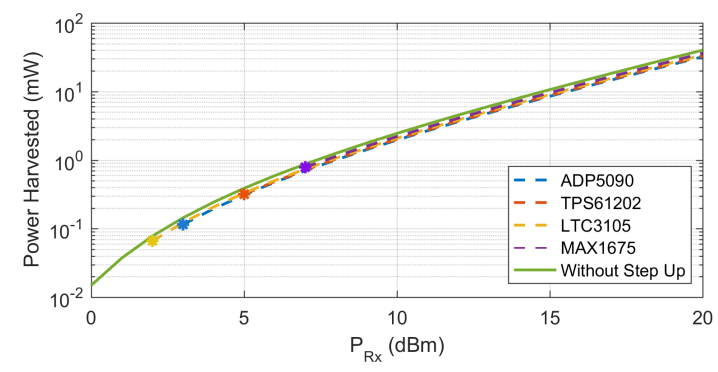

Fig. 5. Simulated power harvested by the rectenna as a function of $P_{R x}$.

The DC-DC boost converter with the best value of $P_{R x}^{\min }$ was the LTC3105, with $2 \mathrm{dBm}$, and it presented the minimum value of start-up voltage among all the converters. Additionally, the difference between the output power of both converters is not significant, e.g. LTC3105 has $34 \mathrm{~mW}$ and MAX1675 has $36 \mathrm{~mW}$ at $20 \mathrm{dBm}$. Therefore, the LTC3105 is selected as the best converter for the designed rectenna and its application in WSN complies with the aforementioned conditions.

\section{EVALUATION OF THE RECTENNA AS ENERGY SOURCE}

In this section, the application of the designed rectenna as energy source is evaluated with different nodes operating at 3.3 V. In that sense, the characteristics of current consumption for the nodes on sleep $\left(I_{\text {sleep }}\right)$ and data transmission $\left(I_{T x}\right)$ of nodes shown in Table II are specified. The node's current consumption $\left(I_{\text {mean }}\right)$ is defined by (4), where $N$ represents the times that the node sends data in one day, $t_{T x}$ is the time to transmit one data packet and receive the acknowledgment packet, and $t_{\text {total }}$ are the seconds in one day.

$$
I_{\text {mean }}=\frac{I_{T x} \cdot t_{T x} \cdot N+I_{\text {sleep }}\left(t_{\text {total }}-t_{T x} \cdot N\right)}{t_{\text {total }}}
$$

TABLE II

CHARACTERISTICS OF THE SELECTED NODES AT AN OPERATING VOLTAGE OF $3.3 \mathrm{~V}$.

\begin{tabular}{|c|c|c|c|}
\hline Node & Band & $I_{\text {sleep }}(\mu \mathbf{A})$ & $I_{T x}(m \mathbf{A})$ \\
\hline CC1350 [13] & Sub-1 GHz & 0.7 & 13.4 \\
\hline CC1350 [13] & $2.4 \mathrm{GHz}$ & 0.7 & 22.3 \\
\hline CC2538 [14] & $2.4 \mathrm{GHz}$ & 1.3 & 24.0 \\
\hline Sky [15] & $2.4 \mathrm{GHz}$ & 54.0 & 19.5 \\
\hline Z1 [16] & $2.4 \mathrm{GHz}$ & 20.5 & 21.4 \\
\hline
\end{tabular}

The evaluated mean current consumption values were 5, 10, 50 and $100 \mu \mathrm{A}$. For the evaluation, $t_{\text {total }}$ equals to $86400 \mathrm{~s}$ and $t_{T x}$ are estimated to be $3 \mathrm{~ms}$ and $9 \mathrm{~ms}$ for the $2.45 \mathrm{GHz}$ and sub-GHz bands, respectively. $I_{T x}$ and $I_{\text {sleep }}$ are those shown in Table II. The Fig. 6 shows the results that allow to identify whether the nodes can send data several times a day with the selected $I_{\text {mean }}$, where $N$ is expressed in packets per hour. Under the studied cases, it was obtained that nodes CC1350 (for both bands) and CC2538 require $5 \mu \mathrm{A}$, node Sky requires $50 \mu \mathrm{A}$, and node $\mathrm{Z} 1$ requires $100 \mu \mathrm{A}$ to operate.

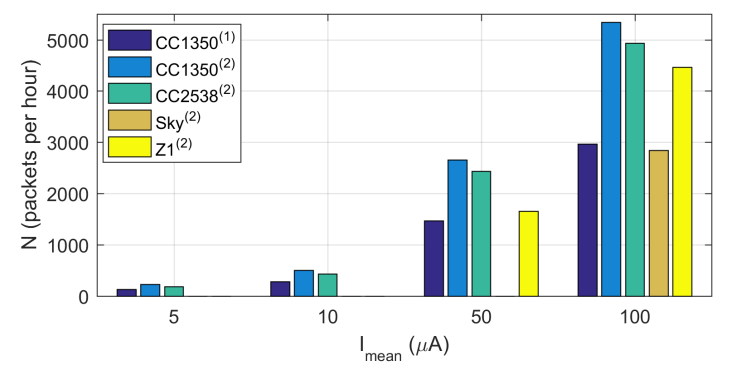

Fig. 6. Packets per hour that the node sends data as function of the mean consumed current for each selected node.

The relationship between the received power and the distance from the transmitting antenna to the rectenna $r$, as a function of the wavelength $\lambda$, is determined by the Friis equation (5), where $G_{T x}$ and $G_{R x}$ are the transmission and reception gains of the antenna, respectively, and $P_{T x}$ is the transmission power. The scenarios for the evaluation are indoor 
and outdoor, the Wi-Fi transmission system for the indoor scenario at $2.45 \mathrm{GHz}$ has $P_{T x}=20 \mathrm{dBm}$ and $G_{T x}=5 \mathrm{dBi}$ [17]. While, the transmission system for the outdoor scenario at $2.45 \mathrm{GHz}$ has $P_{T x}=30 \mathrm{dBm}, G_{T x}=15 \mathrm{dBi}$ [18]. Both scenarios have $G_{R x}=3.38 \mathrm{dBi}$ from the tuned antenna.

$$
P_{R x}=\left(\frac{\lambda}{4 \pi r}\right)^{2} G_{T x} \cdot G_{R x} \cdot P_{T x}
$$

Figure 7 shows the mean power levels required to enable the selected nodes, which are estimated on $16.5 \mu \mathrm{W}$ for CC1350 (in both bands) and CC2538, $165 \mu \mathrm{W}$ for $\mathrm{Z} 1$ and $330 \mu \mathrm{W}$ for Sky. For the nodes CC2538 and CC1350 the power levels needed to operate are under the curve of the power harvested level in both scenarios, hence they can operate along the whole distance range $(0.03 \mathrm{~m}$ to $0.20 \mathrm{~m}$ in indoor scenario and $0.26 \mathrm{~m}$ to $2.03 \mathrm{~m}$ in outdoor scenario).

On the other hand, the Z1 power levels intersects the power harvested curve, reducing the maximum operating distance of the rectenna to $0.17 \mathrm{~m}$ and $1.68 \mathrm{~m}$ for the indoor and outdoor scenarios, respectively. Regarding the Sky node, it also reduces the distance between the rectenna and the wireless transmission source to $0.14 \mathrm{~m}$ and $1.43 \mathrm{~m}$ for the indoor and outdoor scenarios, respectively.

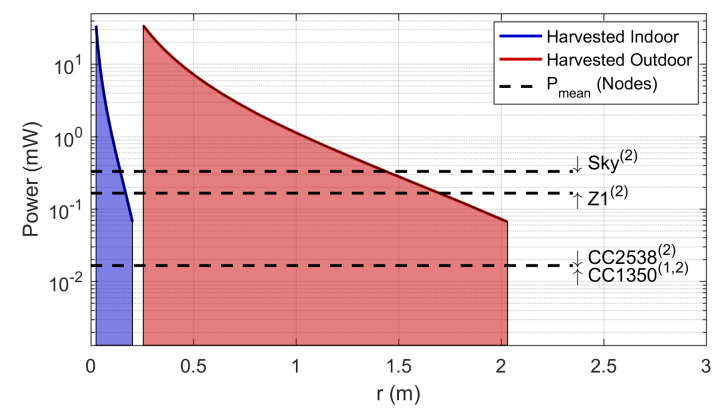

Fig. 7. Power harvested by the rectenna with LTC3105 in indoor and outdoor scenarios and mean consumed power by nodes for the bands Sub-1 GHz (1) and $2.4 \mathrm{GHz}(2)$.

\section{Conclusions}

The conditions for having the rectenna as the only means of energy supply were established. In this case, the node keeps permanently operative without requiring any additional means of energy supply.

The limitations of the designed rectenna were evaluated for two propagation scenarios, indoors and outdoors, using different nodes with diverse technologies. The distance between the rectenna and the wireless energy source that allows the operation at $3.3 \mathrm{~V}$ was estimated. Besides the amount of received power, the minimum input voltage of the DC-DC boost converter proved to be constringent. The distance ranges from $0.03 \mathrm{~m}$ to $0.20 \mathrm{~m}$ in the indoor scenario and from $0.26 \mathrm{~m}$ to $2.03 \mathrm{~m}$ in the outdoor scenario.

For each case scenario, power hungry nodes were able to operate only at the smaller distance within the range, while the most power efficient nodes were able to operate within the whole range. Although the nodes can operate on the estimated distance ranges, these could be improved by modification of the rectenna elements' structure. Future research will be focused on the application of methods to optimize the conversion efficiency and increment the distance ranges.

\section{ACKNOWLEDGMENT}

The authors thank to the Red de Macro Universidades de América Latina y el Caribe (CRAI/DEER/078-12/2018) for contributing to the research cooperation between Universidad del Zulia from Venezuela and Universidad de la República from Uruguay. The authors also thank Rodrigo Enjiu (from CST) for his support with the CST simulation tool.

\section{REFERENCES}

[1] A. Colina, A. Vives, M. Zennaro, A. Bagula, and E. Pietrosemoli, Internet of Things in 5 days, 1st ed., 2016. [Online]. Available: https://github.com/marcozennaro/IPv6-WSN-book

[2] I. F. Akyildiz, W. Su, Y. Sankarasubramaniam, and E. Cayirci, "Wireless sensor networks : a survey," Computer networks, vol. 38, no. 1, pp. 393422, 2002.

[3] J. A. Khan, H. K. Qureshi, and A. Iqbal, "Energy management in Wireless Sensor Networks: A survey," Journal of computers and electrical engineering, vol. 41, pp. 159-176, 2014.

[4] N. Shinohara, "Rectennas for microwave power transmission," IEICE Electronics Express, vol. 10, no. 21, pp. 1-13, 2013.

[5] N. Panda and S. M. Deshmukh, "Novel Technique for Wireless Power Transmission using ISM Band RF Energy Harvesting for Charging Application," International journal of Engineering Trends and Technology (IJETT), vol. 39, no. 2, pp. 63-69, 2016.

[6] C. R. Valenta and G. D. Durgin, "Harvesting Wireless Power," IEEE Microwave Magazine, vol. 15, no. 4, pp. 108-120, 2014.

[7] R. Chakole, M. V. Palandurkar, and M. Renge, "Energy Management of Supercapacitor with DC-DC Converter," in 1st IEEE International Conference on Power Electronics, Intelligent Control and Energy Systems (ICPEICES), Delhi, 2016, pp. 1-6.

[8] CST, "Computer Simulation Technology," 2018. [Online]. Available: https://www.cst.com/

[9] A. Devices, "ADP5090 Ultralow Power Boost Regulator with MPPT and Charge Management," Norwood, pp. 1-21, 2015. [Online]. Available: www.analog.com

[10] T. Instruments, "TPS6120x Low Input Voltage Synchronous Boost Converter With 1.3-A Switches," Texas, pp. 1-35, 2014. [Online]. Available: www.ti.com

[11] L. T. Corporation, "LTC3105 Converter with Maximum Power Point Control and 250mV Start-Up," California, pp. 1-18, 2010. [Online]. Available: www.linear.com/LTC3105

[12] M. integrated, "MAX1674/MAX1675/Max1676 High-Efficiency, LowSupply-Current, Compact, Step-Up DC-DC Converters," California, pp. 1-12, 2000. [Online]. Available: www.maximintegrated.com

[13] T. Instruments, "CC1350 SimpleLink TM Ultra-Low-Power DualBand Wireless MCU," Texas, pp. 1-72, 2018. [Online]. Available: www.ti.com

[14] —, "CC2538 Powerful Wireless Microcontroller System-On-Chip for 2.4-GHz IEEE 802.15.4, 6LoWPAN, and ZigBee® Applications," Texas, pp. 1-34, 2015. [Online]. Available: www.ti.com

[15] Moteiv, "tmote sky. Low Power Wireless Sensor Module," pp. 1-28, 2006. [Online]. Available: https://eva.fing.edu.uy/pluginfile.php/62712/mod_resource/content /1/tmote-Sky-datasheet.pdf

[16] Zolertia, "Z1 Datasheet," pp. 1-20, 2010. [Online]. Available: https://issuu.com/zolertia/docs/z1_datasheet?viewMode=magazine

[17] TP-LINK, " $2.4 \mathrm{GHz} 5 \mathrm{dBi}$ Indoor Omni-directional Antenna. TL ANT2405CL," pp. 1-2. [Online]. Available: www.tp-link.com/no/homenetworking/antenna/tl-ant $2405 \mathrm{cl}$

[18],$- 2.4 \mathrm{~Hz} \quad 15 \mathrm{dBi} \quad$ Outdoor Omni-directional Antenna. TL-ANT2415D," pp. 1-2. [Online]. Available: www.tplink.com/us/products/details/cat-5067_TL-ANT2415D.html\%0A 